\title{
Time-Dependent Characteristics of Two-Layer Functionally Graded Plates Adhesively Bonded by a Viscoelastic Interlayer Based on Kirchhoff Plate Theory
}

\author{
Zhiyuan YANG*, Peng WU**, Weiqing LIU*** \\ *College of Civil Engineering, Nanjing Tech University, Nanjing 211816, China, E-mail: yangzhiyuan@njtech.edu.cn \\ **College of Civil Engineering, Nanjing Tech University, Nanjing 211816, China, E-mail: wupeng2015@yahoo.com \\ ***College of Civil Engineering, Nanjing Tech University, Nanjing 211816, China, E-mail: wqliu@njtech.edu.cn \\ cross $^{\text {ref }}$ http://dx.doi.org/10.5755/j01.mech.26.6.25124
}

\section{Introduction}

Functionally graded (FG) materials composed of two or more constituent phases are being extensively researched due to their continuous property along a particular orientation and are widely applied in weight sensitive areas, such as sensors and actuators, metallic porous and shape memory alloy structure [1-3] etc. Recently, FG material concept was also used in layered plate, including sandwich plates with FG core and/or FG face sheets, to meet the optimal structural design requirements [4-6]. The adjacent layers in layered FG plate are commonly connected by the flexible interlayer made of polymer adhesive with smaller modulus than that of FG layer, which inevitably leads to the slips in the interlayer [7-9]. Moreover, the polymer material naturally possesses the viscoelastic property; therefore, the mechanical behavior of the layered FG plate is actually timedependent and can be greatly influenced by the interlayer [10-12]. The investigation of such a problem becomes essential and deserves in-depth studies.

It is generally known that the analytical solutions can be served as a benchmark to verify the veracity and accuracy of the numerical solutions. Several a nalytical models for the mechanical analysis of layered FG structures have been proposed in literature. Based on the Kirchhoff theory, Moita et al. [13] developed an efficient finite element model to analyze the active-passive damped FG sandwich plates with a viscoelastic core. Joseph and Mohanty [14] designed a finite element (FE) model relied on the first-order shear deformation theory for the analysis of the free vibration of a beam with FG constraining layer and viscoelastic core. By the use of Fourier series and Rayleigh-Ritz method, a unified accurate solution based on the first-order shear deformation theory was provided by Yang et al. [15] to study the vibration and damping of a FG sandwich plate with a soft or hard core. According to the refined zigzag theory, Kolahchi et al. [16] dealt with the general wave propagation in a piezoelectric plate, whose core is consisted of several viscoelastic layers with temperature-dependent behaviors and reinforced by FG carbon nanotubes. Considering the initial geometrical imperfection, Tung [17] studied the bending and postbuckling of FG sandwich plate built upon the first order shear deformation theory by employing the Galerkin procedure. By virtue of the first order shear deformation theory, the electro elastic analysis of the thick-walled FG piezoelectric cylinder was explored by Rahimi et al. [18]. Nejad et al. [19] presented the elastic analysis of the expo- nential FG solid sphere based on the 2-dimensional elasticity theory. A novel quasi-3D shear deformation theory was created for the static and free vibration analysis of FG sandwich plates by Farzam-Rad et al. [20] with the application of the isogeometric analysis method. Furthermore, centered on nth-order shear deformation theory, the natural frequencies of plate with FG face layer and homogeneous core have been studied by Xiang et al. [21] via the meshless global collocation method on account of the thin plate spline radial basis function. Foraboschi [22] proposed an analytical model for two-layer glass plate on the basis of the Kirchhoff plate theory.

However, in the above literatures, the connection between the adjacent layers in structures is considered as perfectly bonded condition or static slip interface, while the long-term response of structures caused by viscoelastic adhesive interlayer is neglected. In the present study, an analytical solution based on the Kirchhoff plate theory for twolayer FG plates bonded by a viscoelastic interlayer is proposed. The mechanical property of interlayer is simulated by the Maxwell-Wiechert model. With the incorporation of Fourier series expansion and energetic method, the potential energy equation of system is obtained and the deformation components can be solved. In addition, the effects of the geometry and material on the time-dependent behaviour of the structure are discussed in detail.

\section{Theoretical model}

The structure investigated is a two-layer plate of length $a$, width $b$ and thickness $H$, comprising two FG facial layers of thickness $h_{i}$ bonded by an adhesive interlayer of thickness $\Delta h$, as shown in Fig. 1, in which the scripts $i$ means the variables belongs to the $i$-th $(i=1,2)$ layer and the variable with superscript * means it belongs to interlayer. $E_{i}(z)$ and $\mu$ denote the elastic modulus and Poisson's ratio of the FG layer, respectively, and $G^{*}(t)$ signifies the shear modulus of the interlayer. $\mu$ holds a constant value in each layer. The plate is subjected to vertical load $q(x, y)$ on its top surface and simply supported at four edges.

\subsection{Assumptions}

Following assumptions are proposed beforehand for the present study:

a) The plate deforms within the range of linearity;

b) The thickness of the adhesive interlayer is small; thus, the interlayer stains are assumed to be constant along the thickness direction; 
c) The adhesive interlayer is rather soft compared with the FG layer; hence, the flexural stiffness in the thin interlayer is ignored.

\subsection{Governing formulations for the FG layer}

The Cartesian coordinate system o-xyz in Fig. 1 is introduced to identify the position of the present structure. Based on the Kirchhoff plate theory, the relations between the deformation components are given by:

$$
u^{i}=u_{0}^{i}-\left(z-z_{i}\right) \frac{\partial w}{\partial x}, v^{i}=v_{0}^{i}-\left(z-z_{i}\right) \frac{\partial w}{\partial y}, i=1,2
$$

in which, $u^{i}, v^{i}$ and $w$ represents the deformations in $x, y$ and $z$ directions, respectively; $z_{i}$ denotes the $z$-coordinate value of the middle plane of the $i$-th layer; $u_{0}^{i}$ and $v_{0}^{i}$ are defined as the deformations of $u^{i}$ and $v^{i}$ on the middle plane, respectively. $u^{i}$ and $v^{i}$ are functions of $x, y, z$ and $t$, while $w$ is the function of $x, y$ and $t$. The geometrical relations can be written as:

$$
\begin{aligned}
& \varepsilon_{x}^{i}=\frac{\partial u_{0}^{i}}{\partial x}-\left(z-z_{i}\right) \frac{\partial^{2} w}{\partial x^{2}}, \varepsilon_{y}^{i}=\frac{\partial v_{0}^{i}}{\partial y}-\left(z-z_{i}\right) \frac{\partial^{2} w}{\partial y^{2}}, \\
& \gamma_{x y}^{i}=\frac{1}{2}\left(\frac{\partial u_{0}^{i}}{\partial y}+\frac{\partial v_{0}^{i}}{\partial x}\right)-\left(z-z_{i}\right) \frac{\partial^{2} w}{\partial x \partial y}, i=1,2
\end{aligned}
$$

where: $\varepsilon_{x}^{i}, \varepsilon_{y}^{i}$ and $\gamma_{x y}^{i}$ are the strains of the $i$-th layer. The constitutive equations in plate are given by:

$$
\left\{\begin{array}{c}
\sigma_{x}^{i} \\
\sigma_{y}^{i} \\
\tau_{x y}^{i}
\end{array}\right\}=\frac{E_{i}(z)}{\left(1-\mu^{2}\right)}\left[\begin{array}{ccc}
1 & \mu & 0 \\
\mu & 1 & 0 \\
0 & 0 & 1-\mu
\end{array}\right]\left\{\begin{array}{c}
\varepsilon_{x}^{i} \\
\varepsilon_{y}^{i} \\
\gamma_{x y}^{i}
\end{array}\right\}, i=1,2 .
$$

By substituting Eq. (2) to Eq. (3), one has:

$$
\begin{aligned}
& \sigma_{x}^{i}=\frac{E_{i}(z)}{\left(1-\mu^{2}\right)}\left[\frac{\partial u_{0}^{i}}{\partial x}+\mu \frac{\partial v_{0}^{i}}{\partial y}-\left(z-z_{i}\right) \times\left(\frac{\partial^{2} w}{\partial x^{2}}+\mu \frac{\partial^{2} w}{\partial y^{2}}\right),\right. \\
& \sigma_{y}^{i}=\frac{E_{i}(z)}{\left(1-\mu^{2}\right)}\left[\mu \frac{\partial u_{0}^{i}}{\partial x}+\frac{\partial v_{0}^{i}}{\partial y}-\left(z-z_{i}\right) \times\left(\mu \frac{\partial^{2} w}{\partial x^{2}}+\frac{\partial^{2} w}{\partial y^{2}}\right),\right. \\
& \tau_{x y}^{i}=\frac{E_{i}(z)}{2(1+\mu)}\left[\frac{\partial u_{0}^{i}}{\partial y}+\frac{\partial v_{0}^{i}}{\partial x}-2\left(z-z_{i}\right) \frac{\partial^{2} w}{\partial x \partial y}\right], i=1,2 .
\end{aligned}
$$

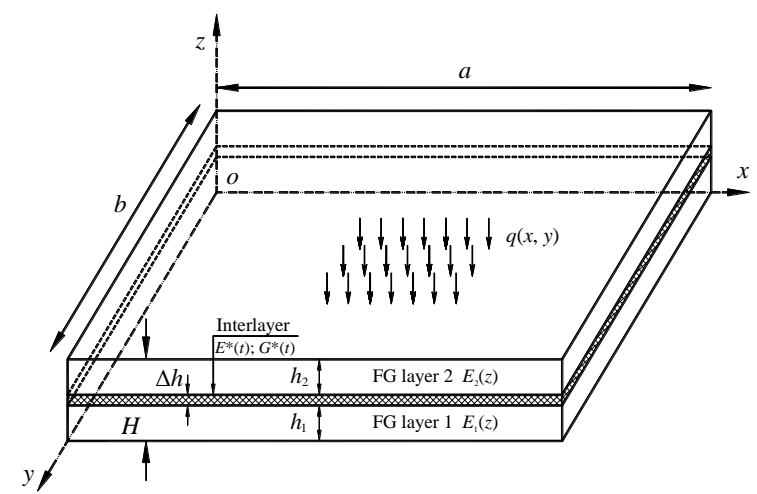

Fig. 1 A two-layer functionally graded plate with viscoelastic interlayer

\subsection{Governing formulations for the interlayer}

The adhesives commonly possess the viscoelastic property, which leads to that the whole system exhibits time-dependent behavior [23]. The Maxwell-Wiechert model, consisting of a series of spring-dashpot units and a spring in parallel as shown in Fig. 2, is adopted for the simulation of the viscoelasticity. Thus, $G^{*}(t)$ can be expressed in the form of Prony series, as follows:

$$
G^{*}(t)=G_{\infty}^{*}+\sum_{j=1}^{n} G_{j}^{*} e^{-t / \theta_{G, j}},
$$

in which, $G_{\infty}^{*}$ means the long-term modulus; $G_{j}^{*}$ denotes the relaxation modulus; $\theta_{G, j}$ represent the relaxation time. These viscoelastic parameters can be achieved by long-term creep tests [24]. As shown in Fig. 3, the geometric equations in the interlayer are given by:

$$
\begin{aligned}
& \gamma_{x z}^{*}=\frac{\left.u^{2}\right|_{z=h_{1}+\Delta h}-\left.u^{1}\right|_{z=h_{1}}}{\Delta h}+\frac{\partial w}{\partial x}, \\
& \gamma_{\mathrm{y} z}^{*}=\frac{\left.v^{2}\right|_{z=h_{1}+\Delta h}-\left.v^{1}\right|_{z=h_{1}}}{\Delta h}+\frac{\partial w}{\partial y} .
\end{aligned}
$$

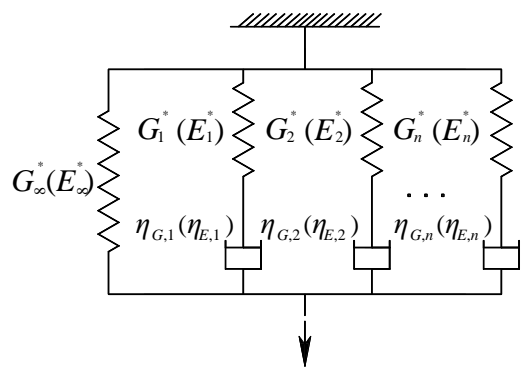

Fig. 2 The Maxwell-Wiechert model

By substituting Eq. (1) into Eq. (6), the shear strains can be rewritten as:

$$
\begin{aligned}
& \gamma_{x z}^{*}=\frac{1}{\Delta h}\left(u_{0}^{2}-u_{0}^{1}+\frac{\partial w}{\partial x} h_{0}\right), \\
& \gamma_{y z}^{*}=\frac{1}{\Delta h}\left(v_{0}^{2}-v_{0}^{1}+\frac{\partial w}{\partial y} h_{0}\right),
\end{aligned}
$$

where: $h_{0}=\left(z_{2}-z_{1}\right)$. According to the Boltzmann superposition principle $[25,26]$, the constitutive equations in the interlayer can be expressed in the form:

$$
\begin{aligned}
& \tau_{x z}^{*}(t)=G^{*}(t) \gamma_{x z}^{*}(0)+\int_{0}^{t} G^{*}(t-\xi) \frac{\partial \gamma_{x z}^{*}(\xi)}{\partial \xi} d \xi, \\
& \tau_{y z}^{*}(t)=G^{*}(t) \gamma_{y z}^{*}(0)+\int_{0}^{t} G^{*}(t-\xi) \frac{\partial \gamma_{y z}^{*}(\xi)}{\partial \xi} d \xi .
\end{aligned}
$$

This means the stress depends on both the current strain and the strain history. However, the calculation considering the strain history is complicated and time-consuming. The quasi-elastic approximation method, which neglects the strain history, can be employed to simplify the viscoelastic constitutive equation. Thus, the stress equations of the viscoelastic interlayer can be reduced as: 


$$
\tau_{x z}^{*}(t)=G^{*}(t) \gamma_{x z}^{*}(t), \tau_{\mathrm{yz}}^{*}(t)=G^{*}(t) \gamma_{y z}^{*}(t)
$$

It should be point out that solution based on the quasi-elastic approximation is always on the side of safety in comparison of the exact solution $[12,27]$.

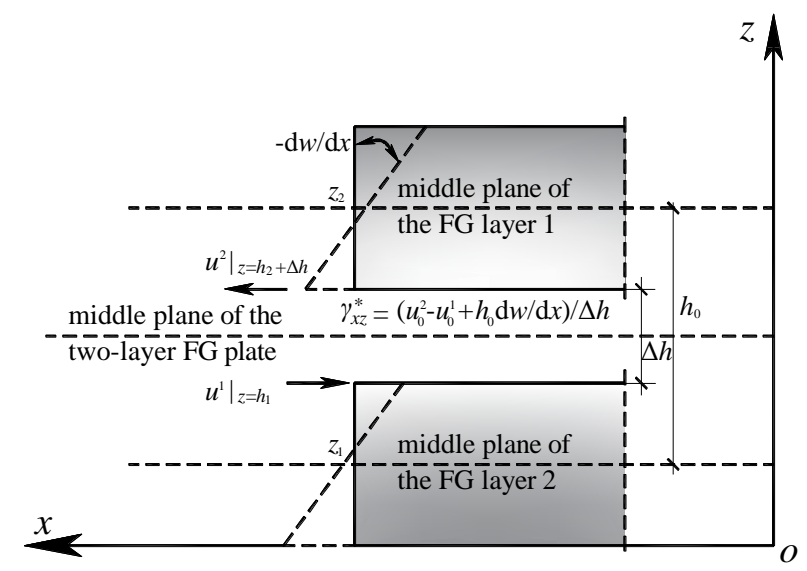

Fig. 3 A deformation profile of an $x$ cross-section

\subsection{The energetic method}

Based on the principle of minimum potential energy, the energy of the system $\Pi$ is expressed as the variational form:

$$
\delta \Pi=\delta U_{1}+\delta U_{2}+\delta U^{*}+\delta V=0,
$$

in which, $\delta$ represents the variation operator and

$$
\begin{aligned}
& U_{1}=\frac{1}{2} \int_{0}^{a} \int_{0}^{b} \int_{0}^{h_{1}}\left(\sigma_{\mathrm{x}}^{1} \varepsilon_{x}^{1}+\tau_{x y}^{1} \gamma_{x y}^{1}\right) d x d y d z, \\
& U_{2}=\frac{1}{2} \int_{0}^{a} \int_{0}^{b} \int_{h_{1}+\Delta h}^{h_{1}+h_{2}+\Delta h}\left(\sigma_{\mathrm{x}}^{2} \varepsilon_{x}^{2}+\tau_{x y}^{2} \gamma_{x y}^{2}\right) d x d y d z, \\
& U^{*}=\frac{1}{2} \int_{0}^{a} \int_{0}^{b} \int_{0}^{\Delta h}\left(\tau_{x z}^{*} \gamma_{x z}^{*}+\tau_{y z}^{*} \gamma_{y z}^{*}\right) d x d y d z, \\
& V=-\int_{0}^{a} \int_{0}^{b} q w d x d y .
\end{aligned}
$$

To simplify the energy functional equation, the extensional stiffness $R_{i}^{e}$, the coupling stiffness $R_{i}^{c}$, and the bending stiffness $R_{i}^{b}$ of each FG layer are respectively defined as follows:

$$
\begin{aligned}
& {\left[\begin{array}{l}
R_{1}^{e} \\
R_{1}^{c} \\
R_{1}^{b}
\end{array}\right]=\int_{0}^{h_{1}} \frac{E_{1}(z)}{\left(1-\mu^{2}\right)}\left[\begin{array}{c}
1 \\
\left(z-z_{1}\right) \\
\left(z-z_{1}\right)^{2}
\end{array}\right] d z,} \\
& {\left[\begin{array}{l}
R_{2}^{e} \\
R_{2}^{c} \\
R_{2}^{b}
\end{array}\right]=\int_{h_{1}+\Delta h}^{H} \frac{E_{2}(z)}{\left(1-\mu^{2}\right)}\left[\begin{array}{c}
1 \\
\left(z-z_{2}\right) \\
\left(z-z_{2}\right)^{2}
\end{array}\right] d z .}
\end{aligned}
$$

has:

By combining Eqs. (2)-(4) with Eqs. (9)-(12), one

$$
\delta \Pi=\int_{0}^{a} \int_{0}^{b} \delta F(x, y, t) d x d y
$$

The corresponding Euler-Lagrange equations of Eq. (13) are given by:

$$
\begin{aligned}
& \frac{\partial F}{\partial u_{0}^{1}}-\frac{\partial}{\partial x} \frac{\partial F}{\partial\left(\partial u_{0}^{1} / \partial x\right)}-\frac{\partial}{\partial y} \frac{\partial F}{\partial\left(\partial u_{0}^{1} / \partial y\right)}=0, \\
& \frac{\partial F}{\partial v_{0}^{1}}-\frac{\partial}{\partial x} \frac{\partial F}{\partial\left(\partial v_{0}^{1} / \partial x\right)}-\frac{\partial}{\partial y} \frac{\partial F}{\partial\left(\partial v_{0}^{1} / \partial y\right)}=0, \\
& \frac{\partial F}{\partial u_{0}^{2}}-\frac{\partial}{\partial x} \frac{\partial F}{\partial\left(\partial u_{0}^{2} / \partial x\right)}-\frac{\partial}{\partial y} \frac{\partial F}{\partial\left(\partial u_{0}^{2} / \partial y\right)}=0, \\
& \frac{\partial F}{\partial v_{0}^{2}}-\frac{\partial}{\partial x} \frac{\partial F}{\partial\left(\partial v_{0}^{2} / \partial x\right)}-\frac{\partial}{\partial y} \frac{\partial F}{\partial\left(\partial v_{0}^{2} / \partial y\right)}=0, \\
& \frac{\partial F}{\partial w}-\frac{\partial}{\partial x} \frac{\partial F}{\partial(\partial w / \partial x)}-\frac{\partial}{\partial y} \frac{\partial F}{\partial(\partial w / \partial y)}+ \\
& +\frac{\partial^{2}}{\partial x^{2}} \frac{\partial^{2} F}{\partial^{2}\left(\partial^{2} w / \partial x^{2}\right)}+\frac{\partial^{2}}{\partial y^{2}} \frac{\partial^{2} F}{\partial^{2}\left(\partial^{2} w / \partial y^{2}\right)}+ \\
& +\frac{\partial^{2}}{\partial x \partial y} \frac{\partial^{2} F}{\partial^{2}\left(\partial^{2} w / \partial x \partial y\right)}=0 .
\end{aligned}
$$

Then, by substituting Eqs. (11-13) into the above equations, one obtains:

$\frac{G^{*}}{\Delta h}\left(u_{0}^{2}-u_{0}^{1}+\frac{\partial w}{\partial x} h_{0}\right)+R_{1}^{e} \frac{\partial^{2} u_{0}^{1}}{\partial x^{2}}+\frac{(1-\mu)}{2} R_{1}^{e} \frac{\partial^{2} u_{0}^{1}}{\partial y^{2}}-$

$-R_{1}^{c} \frac{\partial^{3} w}{\partial x^{3}}-R_{1}^{c} \frac{\partial^{3} w}{\partial x \partial y^{2}}=0+\frac{(1+\mu)}{2} R_{1}^{e} \frac{\partial^{2} v_{0}^{1}}{\partial x \partial y}$,

$\frac{G^{*}}{\Delta h}\left(v_{0}^{2}-v_{0}^{1}+\frac{\partial w}{\partial y} h_{0}\right)+\frac{(1+\mu)}{2} R_{1}^{e} \frac{\partial^{2} u_{0}^{1}}{\partial x \partial y}+$

$+\frac{(1-\mu)}{2} R_{1}^{e} \frac{\partial^{2} v_{0}^{1}}{\partial x^{2}}+R_{1}^{e} \frac{\partial^{2} v_{0}^{1}}{\partial y^{2}}-R_{1}^{c} \frac{\partial^{3} w}{\partial y^{3}}-R_{1}^{c} \frac{\partial^{3} w}{\partial x^{2} \partial y}=0$,

$\frac{G^{*}}{\Delta h}\left(u_{0}^{2}-u_{0}^{1}+\frac{\partial w}{\partial x} h_{0}\right)-R_{2}^{e} \frac{\partial^{2} u_{0}^{2}}{\partial x^{2}}-\frac{(1-\mu)}{2} R_{2}^{e} \frac{\partial^{2} u_{0}^{2}}{\partial y^{2}}-$

$-\frac{(1+\mu)}{2} R_{2}^{e} \frac{\partial^{2} v_{0}^{2}}{\partial x \partial y}+R_{2}^{c} \frac{\partial^{3} w}{\partial x^{3}}+R_{2}^{c} \frac{\partial^{3} w}{\partial x \partial y^{2}}=0$,

$\frac{G^{*}}{\Delta h}\left(v_{0}^{2}-v_{0}^{1}+\frac{\partial w}{\partial y} h_{0}\right)-\frac{(1+\mu)}{2} R_{2}^{e} \frac{\partial^{2} u_{0}^{2}}{\partial x \partial y}-R_{2}^{e} \frac{\partial^{2} v_{0}^{2}}{\partial y^{2}}-$

$-\frac{(1-\mu)}{2} R_{2}^{e} \frac{\partial^{2} v_{0}^{2}}{\partial x^{2}}+R_{2}^{c} \frac{\partial^{3} w}{\partial y^{3}}+R_{2}^{c} \frac{\partial^{3} w}{\partial x^{2} \partial y}=0$,

$\frac{G^{*} h_{0}}{\Delta h}\left(\frac{\partial u_{0}^{2}}{\partial x}-\frac{\partial u_{0}^{1}}{\partial x}+\frac{\partial^{2} w}{\partial x^{2}} h_{0}\right)+R_{1}^{c} \frac{\partial^{3} u_{0}^{1}}{\partial x^{3}}+R_{2}^{c} \frac{\partial^{3} u_{0}^{2}}{\partial x^{3}}+$

$+\frac{G^{*} h_{0}}{\Delta h}\left(\frac{\partial v_{0}^{2}}{\partial y}-\frac{\partial v_{0}^{1}}{\partial y}+\frac{\partial^{2} w}{\partial y^{2}} h_{0}\right)+R_{2}^{c} \frac{\partial^{3} u_{0}^{2}}{\partial x \partial y^{2}}+$

$+R_{1}^{c} \frac{\partial^{3} v_{0}^{1}}{\partial y^{3}}+R_{2}^{c} \frac{\partial^{3} v_{0}^{2}}{\partial y^{3}}+R_{1}^{c} \frac{\partial^{3} u_{0}^{1}}{\partial x \partial y^{2}}+R_{1}^{c} \frac{\partial^{3} v_{0}^{1}}{\partial x^{2} \partial y}+$

$+R_{2}^{c} \frac{\partial^{3} v_{0}^{2}}{\partial x^{2} \partial y}-\left(R_{1}^{b}+R_{2}^{b}\right) \frac{\partial^{4} w}{\partial x^{4}}-\left(R_{1}^{b}+R_{2}^{b}\right) \frac{\partial^{4} w}{\partial y^{4}}-$

$-2\left(R_{1}^{b}+R_{2}^{b}\right) \frac{\partial^{4} w}{\partial x^{2} \partial y^{2}}+q(x, y, t)=0$.

The boundary conditions of the simply supported layered FG plate are given by: 


$$
\begin{aligned}
\sigma_{x}^{i} & =v=w=0 \text { at } x=0, a ; \\
\sigma_{y}^{i} & =u=w=0 \text { at } y=0, b .
\end{aligned}
$$

Resorting to the Navier's method, the applied load and the deformations are expanded as the form of double trigonometric series:

$$
\left[\begin{array}{c}
u_{0}^{1} \\
u_{0}^{2} \\
v_{0}^{1} \\
v_{0}^{2} \\
w \\
q
\end{array}\right]=\sum_{m=1}^{\infty} \sum_{n=1}^{\infty}\left[\begin{array}{l}
u_{1}^{m n}(t) \cos \left(\alpha_{m} x\right) \sin \left(\beta_{n} y\right) \\
u_{2}^{m n}(t) \cos \left(\alpha_{m} x\right) \sin \left(\beta_{n} y\right) \\
v_{1}^{m n}(t) \sin \left(\alpha_{m} x\right) \cos \left(\beta_{n} y\right) \\
v_{2}^{m n}(t) \sin \left(\alpha_{m} x\right) \cos \left(\beta_{n} y\right) \\
w^{m n}(t) \sin \left(\alpha_{m} x\right) \sin \left(\beta_{n} y\right) \\
q^{m n}(t) \sin \left(\alpha_{m} x\right) \sin \left(\beta_{n} y\right)
\end{array}\right],
$$

in which, $\alpha_{m}=m \pi / a, \beta_{n}=n \pi / b, q^{m n}(t)=$ $=-\frac{4}{a b} \int_{0}^{a} \int_{0}^{b} q(x, y, t) \sin \left(\alpha_{m} x\right) \sin \left(\beta_{n} y\right) d x d y$.

By substituting Eq. (17) into Eq. (15), the unknown variables can be obtained by the Cramer's Rule and given by as follows:

$$
\left[\begin{array}{c}
u_{1}^{m n}(t) \\
u_{2}^{m n}(t) \\
v_{1}^{m n}(t) \\
v_{2}^{m n}(t) \\
w^{m n}(t)
\end{array}\right]=-\frac{q^{m n}(t)}{\varphi_{m n}^{4}}\left[\begin{array}{c}
\alpha_{m} \frac{\varphi_{m n}^{2} p_{1}+p_{2} G^{*}(t)}{\varphi_{m n}^{2} r_{1}+r_{2} G^{*}(t)} \\
\alpha_{m} \frac{\varphi_{m n}^{2} p_{3}+p_{4} G^{*}(t)}{\varphi_{m n}^{2} r_{3}+r_{4} G^{*}(t)} \\
\beta_{n} \frac{\varphi_{m n}^{2} p_{1}+p_{2} G^{*}(t)}{\varphi_{m n}^{2} r_{1}+r_{2} G^{*}(t)} \\
\beta_{n} \frac{\varphi_{m n}^{2} p_{3}+p_{4} G^{*}(t)}{\varphi_{m n}^{2} r_{3}+r_{4} G^{*}(t)} \\
\frac{\varphi_{m n}^{2} p_{5}+p_{6} G^{*}(t)}{\varphi_{m n}^{2} r_{3}+r_{4} G^{*}(t)}
\end{array}\right],
$$

where: $\varphi_{\mathrm{mn}}=\sqrt{\alpha_{m}^{2}+\beta_{n}^{2}}, p_{1}=\Delta h R_{2}^{e} R_{1}^{c}$,

$$
\begin{aligned}
& p_{2}=h_{0} R_{2}^{e}+R_{1}^{c}+R_{1}^{c}, p_{3}=\Delta h R_{1}^{e} R_{2}^{c}, \\
& p_{4}=-h_{0} R_{1}^{e}+R_{1}^{c}+R_{2}^{c}, p_{5}=\Delta h R_{1}^{e} R_{2}^{e}, \\
& p_{6}=R_{1}^{e}+R_{2}^{e}, \\
& r_{1}=\Delta h\left[-R_{1}^{e} R_{2}^{e}\left(R_{1}^{b}+R_{2}^{b}\right)+R_{2}^{e}\left(R_{1}^{c}\right)^{2}\right]+\Delta h R_{1}^{e}\left(R_{2}^{c}\right)^{2}, \\
& r_{2}=\left(R_{1}^{c}+R_{2}^{c}\right)^{2}+R_{1}^{e}\left(-2 h_{0} R_{2}^{c}-R_{1}^{b}-R_{2}^{b}\right)+ \\
& +R_{2}^{e}\left(-h_{0}^{2} R_{1}^{e}+2 h_{0} R_{1}^{c}-R_{1}^{b}-R_{2}^{b}\right), \\
& r_{3}=\Delta h\left[-R_{1}^{e} R_{2}^{e}\left(R_{1}^{b}+R_{2}^{b}\right)+R_{1}^{e}\left(R_{2}^{c}\right)^{2}\right]+\Delta h R_{2}^{e}\left(R_{1}^{c}\right)^{2}, \\
& r_{4}=R_{1}^{e}\left(-h_{0}^{2} R_{2}^{e}-2 h_{0} R_{2}^{c}-R_{1}^{b}-R_{2}^{b}\right)+ \\
& \left(R_{1}^{c}+R_{2}^{c}\right)^{2}+R_{2}^{e}\left(2 h_{0} R_{1}^{c}-R_{1}^{b}-R_{2}^{b}\right) .
\end{aligned}
$$

By substitution of the determined variables in Eq. (18) back into Eqs. (17), (1) and (4), respectively, the analytical solutions of stress and deformation can be obtained eventually.

\section{Numerical examples and discussion}

In this section, a FG system with two constituents, formed by metal and ceramic materials, is considered, and their volume fractions are rendered as the power-law relations through the $z$ direction, which are respectively defined by:

$$
\begin{aligned}
& V_{m, 1}(z)=\left\{\begin{array}{ll}
1-\left(z / h_{1}\right)^{k_{1}} & \text { for } k_{1} \geq 1 \\
{\left[1-\left(z / h_{1}\right)\right]^{1 / k_{1}}} & \text { for } k_{1} \leq 1
\end{array},\right. \\
& V_{m, 2}(z)= \begin{cases}1-\left[(H-z) / h_{2}\right]^{k_{2}} & \text { for } k_{2} \geq 1 \\
\left\{1-\left[(H-z) / h_{2}\right]\right\}^{1 / k_{2}} \text { for } k_{2} \leq 1\end{cases} \\
& V_{c, 1}(z)=1-V_{m, 1}(z), \\
& V_{c, 2}(z)=1-V_{m, 2}(z),
\end{aligned}
$$

in which, the scripts $m$ and $c$ represent the variables belonging to the metal and ceramic layers, respectively; $k_{i}$ is nonnegative real number named as gradient factor. The layer degenerates to a metal case when $k_{i} \rightarrow \infty$, while it degenerates to a ceramic case as $k_{i} \rightarrow 0$. The elastic moduli in FG layers are determined by the modified rule of mixtures [28], as follows:

$$
E_{\mathrm{i}}(z)=\frac{E_{m} V_{m, i}(z)\left(\phi+E_{c}\right)+E_{c} V_{c, i}(z)\left(\phi+E_{m}\right)}{V_{m, i}(z)\left(\phi+E_{c}\right)+V_{c, i}(z)\left(\phi+E_{m}\right)},
$$

where: $\phi$ means the ratio of stress to strain transferring between the two phases which can be achieved by material test $[29,30]$. The typical metal-ceramic $\mathrm{FG}$ system $\mathrm{Al} / \mathrm{SiC}$ is chosen to be analyzed in this part and the basic properties of this system are $E_{m}=67 \mathrm{GPa}, E_{c}=302 \mathrm{GPa}, \phi=91.6 \mathrm{GPa}$ and $\mu_{m}=\mu_{c}=0.25$ [29].

Eight variables are beforehand defined for the following analysis: $\sigma_{x}^{l}, \sigma_{y}^{l}, w^{l}$ represent $\sigma_{x}^{1}, \sigma_{y}^{1}$ and $w$ at $x=0.5 a, y=0.5 b, z=0$ respectively; $\tau_{x y}^{r}, u^{r}$ and $v^{r}$ denote $\tau_{x y}^{1}, u^{1}$ and $v^{1}$ at $x=0.25 a, y=0.25 b, z=h_{1}$ respectively; $\tau_{x z}^{* r}$ and $\tau_{y z}^{* r}$ are $\tau_{x z}^{*}$ and $\tau_{y z}^{*}$ at $x=0.25 a, y=0.25 b$, respectively. The variable with two external vertical bar denotes its absolute value, e.g., $|w|$; meanwhile the variable with subscript max represents its maximum value, e.g., $w_{\max }$.

\subsection{Validation analysis}

In this part, the $\mathrm{Al} / \mathrm{SiC}$ system is applied in $\mathrm{FG}$ layers and the shear relaxation moduli of the PVB as the interlayer are listed in Table 1. The analytical solutions are compared with the FE solutions which are given by the commercial software ANSYS. The material of interlayer is simulated by the VISCO-89 elements. Since ANSYS cannot directly model the FG material, the two FG layers here are equally divided into $\lambda$ isotropic sub-layers with each modeled by the PLANE-183 element. The elastic modulus in each sub-layer is determined by $E_{i}\left(z_{i}^{\lambda}\right)$, in which $z_{i}^{\lambda}$ represents the z-coordinate of the middle plane of each sublayer. The FG layer and interlayer in $x-y$ plane are divided by $\lambda \times \lambda$ parts. The geometric and material parameters of the plate are taken as $a=1000 \mathrm{~mm}, H=20 \mathrm{~mm}, k_{1}=k_{2}$. 
Table 1

The relaxation moduli and relaxation time in the viscoelastic interlayer (PVB)

\begin{tabular}{|c|c|c|}
\hline$j$ & $G_{j}^{*}, \mathrm{MPa}$ & $\theta_{G, j}, \mathrm{~s}$ \\
\hline 1 & 75.6426 & $3.256 \times 10^{-11}$ \\
\hline 2 & 37.0677 & $4.949 \times 10^{-9}$ \\
\hline 3 & 137.1552 & $7.243 \times 10^{-8}$ \\
\hline 4 & 33.5140 & $9.864 \times 10^{-6}$ \\
\hline 5 & 126.6048 & $2.806 \times 10^{-3}$ \\
\hline 6 & 42.1950 & $1.644 \times 10^{-1}$ \\
\hline 7 & 14.2162 & $2.265 \times 10^{0}$ \\
\hline 8 & 3.5822 & $3.536 \times 10^{1}$ \\
\hline 9 & 0.4538 & $9.368 \times 10^{3}$ \\
\hline 10 & 0.1912 & $6.414 \times 10^{5}$ \\
\hline 11 & 0.2893 & $4.135 \times 10^{7}$ \\
\hline$\infty$ & 0.0880 & \\
\hline
\end{tabular}

Table 2 displays the comparison of $\sigma_{x}^{l}, \tau_{x z}^{{ }^{*} r}$ and $w^{l}$ between the present results and FE ones with $k_{1}=3$ and $t=10^{4} \mathrm{~s}$ for different $\lambda$ and different length-width ratios $a / b$, respectively. It can be observed from Table 2 that a good agreement is obtained between the present and FE results as $\lambda$ increases, and the relative errors of stresses and deformations are less than $2.25 \%$ when $\lambda=20$. It is worth emphasizing that the precise FE solutions are highly time-consuming to be obtained because of the fine mesh both in geometric shape and the time step.

\subsection{Parametric analysis}

Consider a simply supported layered $\mathrm{Al} / \mathrm{SiC} \mathrm{FG}$ plate subjected to a sinusoidal load $q(x, y)=$ $=\sin (\pi x / a) \sin (\pi y / b) \mathrm{N} / \mathrm{mm}^{2}$ on its top surface. The geometric parameters are fixed at $a=800 \mathrm{~mm}, b=1000 \mathrm{~mm}$, $\Delta h=0.2 \mathrm{~mm}$.
Table 2

A comparison of $\sigma_{x}^{l}, \tau_{x z}^{* r}$ and $w^{l}$ between the present results and the FE results for different gradient factor and length-width ratio

\begin{tabular}{|c|c|c|c|c|c|c|}
\hline \multirow{2}{*}{$a / b$} & \multirow{2}{*}{ solution } & \multirow{2}{*}{ present } & \multicolumn{4}{|c|}{$\bar{\lambda}$} \\
\hline & & & 1 & 5 & 10 & 20 \\
\hline \multirow{6}{*}{2} & $\sigma_{\mathrm{x}}^{l}, \mathrm{MPa}$ & 163.9 & 193.2 & 168.3 & 165.8 & 165.1 \\
\hline & Error, \% & 1 & 15.2 & 2.62 & 1.15 & 0.72 \\
\hline & $\tau_{x z}^{* r}, \mathrm{MPa}$ & -0.645 & -0.950 & -0.696 & -0.665 & -0.657 \\
\hline & Error, \% & 1 & 32.2 & 7.39 & 3.11 & 1.81 \\
\hline & $w^{l}, \mathrm{~mm}$ & -17.43 & -20.14 & -18.21 & -17.76 & -17.63 \\
\hline & Error, \% & 1 & 13.43 & 4.26 & 1.87 & 1.14 \\
\hline \multirow{6}{*}{4} & $\sigma_{\mathrm{x}}^{l}, \mathrm{MPa}$ & 37.95 & 47.69 & 38.91 & 38.07 & 37.83 \\
\hline & Error, \% & 1 & 20.4 & 2.47 & 0.32 & 0.31 \\
\hline & $\tau_{x z}^{* r}, \mathrm{MPa}$ & -0.068 & -0.115 & -0.074 & -0.070 & -0.069 \\
\hline & Error, \% & 1 & 41.1 & 8.97 & 3.81 & 2.25 \\
\hline & $w^{l}, \mathrm{~mm}$ & -1.688 & -2.175 & -1.771 & -1.712 & -1.695 \\
\hline & Error, \% & 1 & 22.4 & 4.68 & 1.40 & 0.43 \\
\hline
\end{tabular}

The distributions of stresses and deformations in structure with $k_{1}=k_{2}=1 / 3$ when $t=10 \mathrm{~s}, 10^{2} \mathrm{~s}, 10^{5} \mathrm{~s}$ are presented in Fig. 4, in which PB means the perfectly bonded case. It can be observed from Fig. 4 that $\left|\sigma_{x}^{i}\right|$ and $\left|u^{i}\right|$ close to the surfaces and the interlayer increases with $t$, and $|w|$ increases with $t .\left|\tau_{x z}^{*}\right|_{\max }$ in $x-y$ plane decreases with $t$, while $\left|\tau_{x y}^{i}\right|$ in $x-y$ plane increases with $t$. Compared with the PB case, $\left|\sigma_{x}^{i}\right|_{\max },\left|u^{i}\right|_{\max },|w|_{\max }$, and $\left|\tau_{x y}\right|_{\max }$ increases by $157.3 \%, 82.3 \%, 191.7 \%, 82.4 \%$, respectively when $t=10^{5} \mathrm{~s}$, while $\left|\tau_{x z}^{*}\right|_{\max }$ decreases by $77.1 \%$.

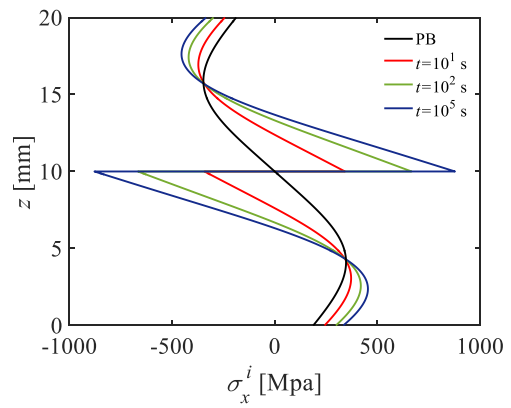

a) $\sigma_{x}^{i}(x=400 \mathrm{~mm}, y=500 \mathrm{~mm})$

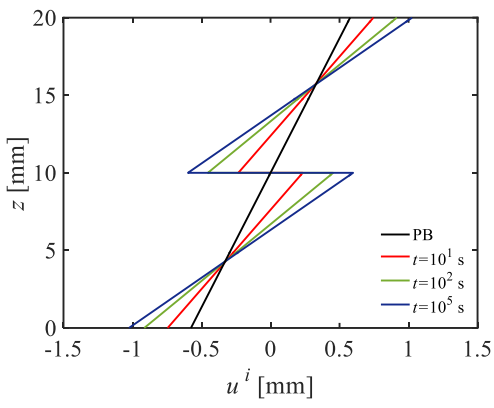

b) $u^{i}(x=0 \mathrm{~mm}, y=500 \mathrm{~mm})$

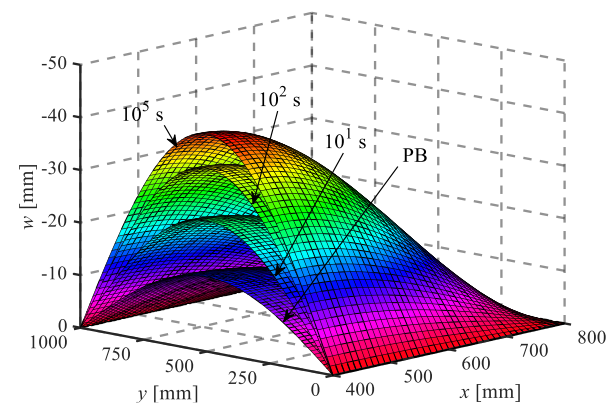

c) $w$

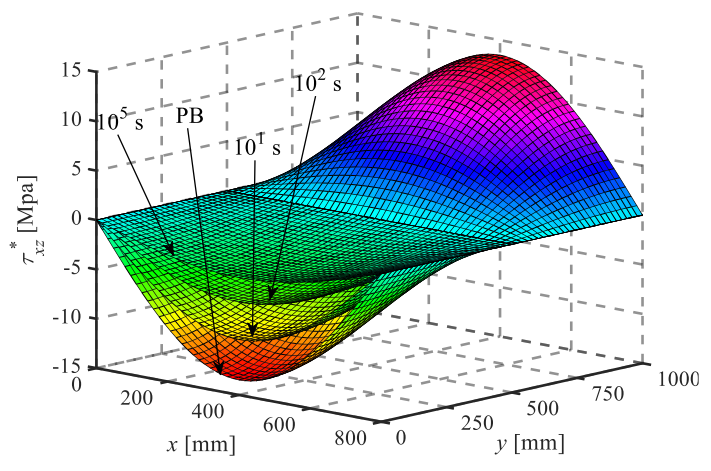

d) $\tau_{x z}^{*}$

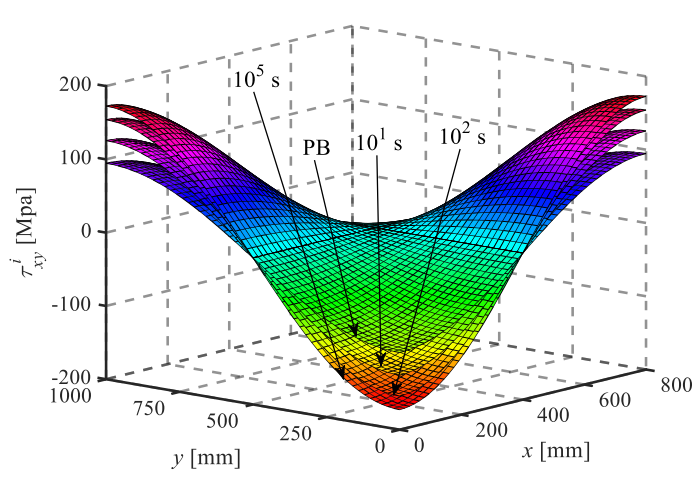

e) $\tau_{x y}^{i}(z=0 \mathrm{~mm})$

Fig. 4 Distributions of the stress and deformation components and those corresponding PB cases when $t=10 \mathrm{~s}, 10^{2} \mathrm{~s}$ and $10^{5} \mathrm{~s}$, respectively when $k_{1}=k_{2}=1 / 3$ 
The influences of $k$ and $\Delta h$ on $\sigma_{x}^{l}, \tau_{x z}^{* r}, w^{l}$ in model III are presented in Fig. 5. It can be found from Fig. 5 that for a given $\Delta h,\left|\sigma_{x}^{l}\right|$ and $\left|w^{l}\right|$ increase with $t$ and reach at a fixed value as $t$ is close to $10^{10} \mathrm{~s}$, while $\left|\tau_{x z}^{* r}\right|$ decreases with $t$ and is reduced to a constant as $t$ draws near $10^{10} \mathrm{~s}$. The rise of the $\Delta h$ leads to an obvious increase of $\left|\sigma_{x}^{l}\right|$ and $\left|w^{l}\right|$ at any $t$, while $\left|\tau_{x z}^{* r}\right|$ exhibits a downward trend as $\Delta h$ increases at any $t$. For a given $k,\left|\sigma_{x}^{l}\right|$ and $\left|w^{l}\right|$

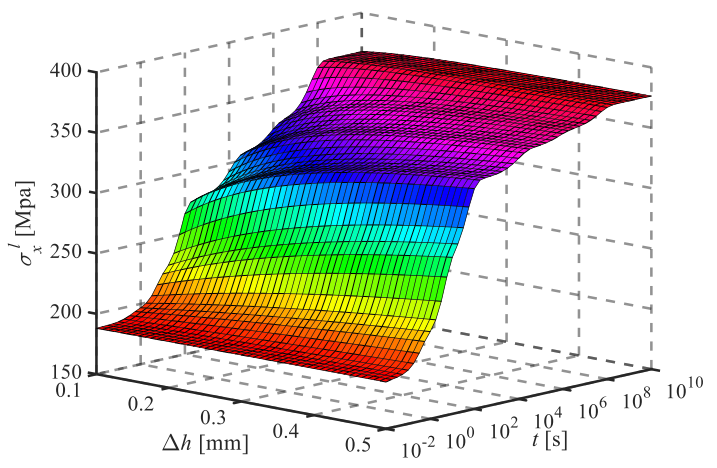

a) $\sigma_{x}^{l}(k=1 / 3)$

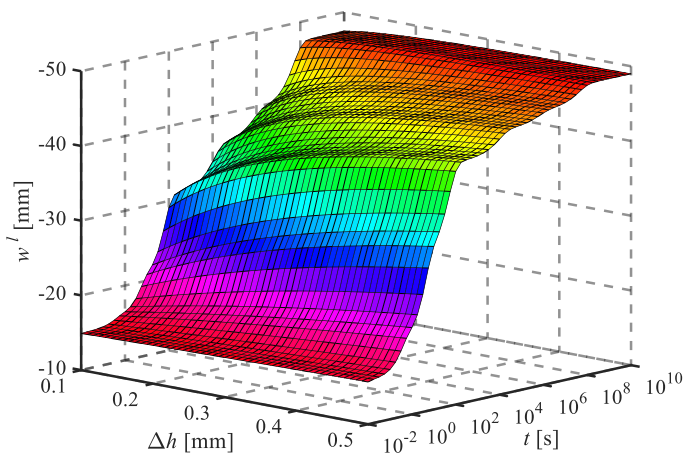

c) $w^{l}(k=1 / 3)$

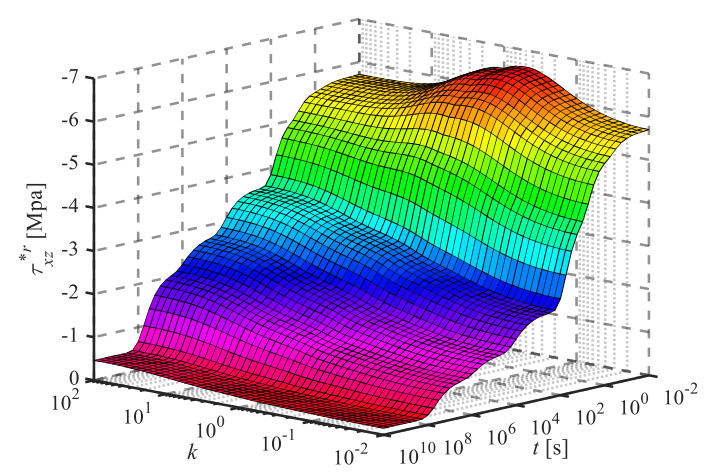

e) $\tau_{x z}^{* r}(\Delta h=0.2 \mathrm{~mm})$ increase with $t$ and reach at a fixed value as $t$ is close to $10^{10}$ s. Similarly, $\left|\sigma_{x}^{l}\right|$ and $\left|w^{l}\right|$ increase with the rise of $k$ and tend to be constants at any $t$. For a given $k,\left|\tau_{x z}^{* r}\right|$ monotonically decreases with $t$ and tend to be a constant as $t$ draws near $10^{10} \mathrm{~s}$. In early stage, $\left|\tau_{x z}^{* r}\right|$ increases initially and then decreases with the rise of $k$, while $\left|\tau_{x z}^{* r}\right|$ monotonically goes up as $k$ increases when $t>10^{3} \mathrm{~s}$.

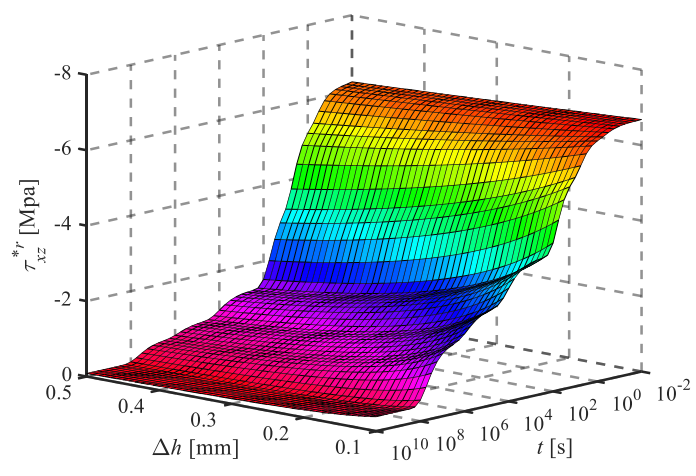

b) $\tau_{x z}^{* r}(k=1 / 3)$

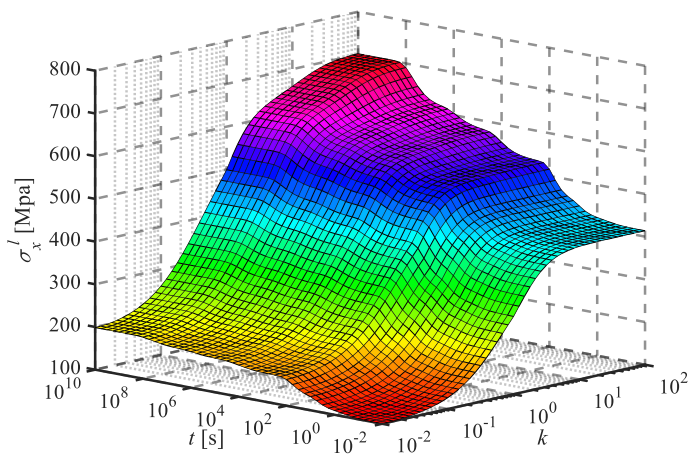

d) $\sigma_{x}^{l}(\Delta h=0.2 \mathrm{~mm})$

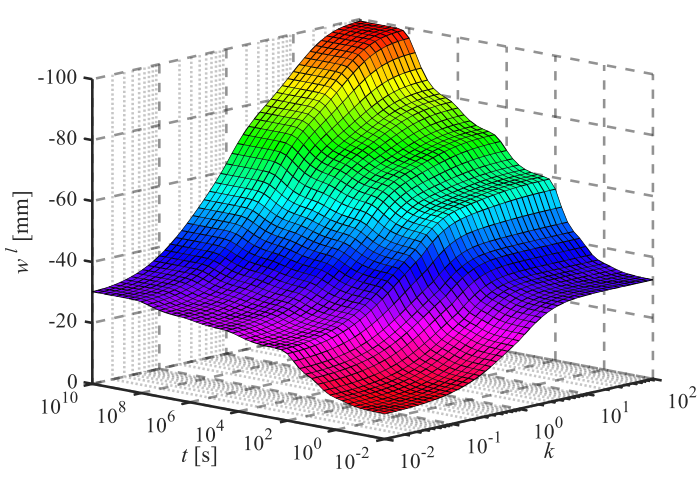

f) $w^{l}(\Delta h=0.2 \mathrm{~mm})$

Fig. 5 Variations of $\sigma_{x}^{l}, \tau_{x z}^{* r}$ and $w^{l}$ with time for different gradient factor and different interlayer thickness

\section{Conclusions}

In the present work, an analytical solution based on the Kirchhoff plate theory is proposed to analyze the timedependent behaviors of the two-layer FG plate with adhesive interlayer. The obtained results provide the following conclusions:
1. The FE results are in great agreement with the present one. However, the FE solutions are highly time-consuming to be obtained because of the fine mesh both in geometric shape and the time step.

2. The longitudinal stress, longitudinal displacement and deflection increase with time, while the shear stress in 
the interlayer decreases with time. In contrast to the perfectly bonded case, the maximum value of deflection increases by $191.7 \%$ and that of shear stress decreases by $77.1 \%$.

3. The stress and deformation both tend to be constant in the long term. The increase of the thickness of the interlayer leads to the growth of the longitudinal stress and deflection, while the shear stress in the interlayer decreases as the interlayer thickness increases.

4. The longitudinal stress and deflection both show an upward trend as gradient factor increases and remain unchanged at any time. However, with the rise of gradient factor, the shear stress increases initially and then decreases to a constant in early stage, while in the medium term it has a monotonic rise and then stays the same as gradient factor increases.

\section{Acknowledgements}

This research is financially supported by the National Natural Science Foundation of China (Grant No. 51778285), the Natural Science Foundation of Jiangsu Province (Grant No. BK20190668) and the Natural Science Foundation of the Jiangsu Higher Education Institutions of China (Grant No. 19KJB560014).

\section{References}

1. Alibeigloo, A.; Simintan, V. 2011. Elasticity solution of functionally graded circular and annular plates integrated with sensor and actuator layers using differential quadrature, Composite Structures 93: 2473-2486. https://doi.org/10.1016/j.compstruct.2011.04.003.

2. Gao, K.; Huang, Q.; Kitipornchai, Q. 2019. Nonlinear dynamic buckling of functionally graded porous beams, Mechanics of Advanced Materials and Structures: 1-12. https://doi.org/10.1080/15376494.2019.1567888.

3. Asadi, H.; Akbarzadeh, A. H.; Wang, Q. 2015. Nonlinear thermo-inertial instability of functionally graded shape memory alloy sandwich plates, Composite Structures 120: 496-508. https://doi.org/10.1016/j.compstruct.2014.10.027.

4. Liu, M.; Cheng, Y. S.; Liu, J. 2015. High-order free vibration analysis of sandwich plates with both functionally graded face sheets and functionally graded flexible core, Composites Part B-Engineering 72: 97-107. https://doi.org/10.1016/j.compositesb.2014.11.037.

5. Merdaci, S.; Tounsi, A.; Houari, M. S. A.; Mechab, I.; Hebali, H.; Benyoucef, S. 2011. Two new refined shear displacement models for functionally graded sandwich plates, Archive of Applied Mechanics 81: 1507-1522. https://doi.org/10.1007/s00419-010-0497-5.

6. Ganapathi, M.; Anirudh, B.; Anant, C.; Polit, O. 2019. Dynamic characteristics of functionally graded graphene reinforced porous nanocomposite curved beams based on trigonometric shear deformation theory with thickness stretch effect, Mechanics of Advanced Materials and Structures: 1-12. https://doi.org/10.1080/15376494.2019.1601310.

7. Ecsedi, I.; Baksa, A. 2011. Static analysis of composite beams with weak shear connection, Applied Mathematical Modelling 35 (4): 1739-1750. https://doi.org/10.1016/j.apm.2010.10.006.

8. Kwak, H. G.; Seo, Y. J. 2002. Time-dependent behavior of composite beams with flexible connectors. Computer Methods in Applied Mechanics and Engineering 191 (34): 3751-3772.

https://doi.org/10.1016/s0045-7825(02)00293-1.

9. Foraboschi, P. 2009. Analytical solution of two-layer beam taking into account nonlinear interlayer slip, Journal of Engineering Mechanics 135 (10): 1129-1146. https://doi.org/10.1061/(asce)em.1943-7889.0000043.

10. Ranzi, G.; Bradford, M. 2006. Analytical solutions for the time-dependent behaviour of composite beams with partial interaction, International Journal of Solids and Structures 43 (13): 3770-3793.

https://doi.org/10.1016/j.ijsolstr.2005.03.032.

11. Chen, W. Q.; Lee, K. Y. 2004. Time-dependent behaviors of angle-ply laminates with viscous interfaces in cylindrical bending, European Journal of Mechanics a-Solids 23 (2): 235-245. https://doi.org/10.1016/j.euromechsol.2003.12.004.

12. Galuppi, L.; Royer-Carfagni, G. 2012. Laminated beams with viscoelastic interlayer, International Journal of Solids and Structures 49 (18): 2637-2645. https://doi.org/10.1016/j.ijsolstr.2012.05.028.

13. Moita, J. S.; Araujo, A. L.; Correia, V. F.; Soares, C. M. M.; Herskovits, J. 2018. Active-passive damping in functionally graded sandwich plate/shell structures, Composite Structures 202: 324-332.

https://doi.org/10.1016/j.compstruct.2018.01.089.

14. Joseph, S. V.; Mohanty, S. C. 2017. Free vibration of a rotating sandwich plate with viscoelastic core and functionally graded material constraining layer, International Journal of Structural Stability and Dynamics 17 (10): 1750114. https://doi.org/10.1142/s0219455417501140.

15. Yang, C. M.; Jin, G. Y.; Ye, X. M.; Liu, Z. G. 2016. A modified Fourier-Ritz solution for vibration and damping analysis of sandwich plates with viscoelastic and functionally graded materials, International Journal of Mechanical Sciences 106: 1-18.

https://doi.org/10.1016/j.ijmecsci.2015.11.031.

16. Kolahchi, R.; Zarei, M. S.; Hajmohammad, M. H.; Nouri, A. 2017. Wave propagation of embedded viscoelastic FG-CNT-reinforced sandwich plates integrated with sensor and actuator based on refined zigzag theory, International Journal of Mechanical Sciences 130: 534545 . https://doi.org/10.1016/j.ijmecsci.2017.06.039.

17. Tung, H. V. 2015. Thermal and thermomechanical postbuckling of FGM sandwich plates resting on elastic foundations with tangential edge constraints and temperature dependent properties, Composite Structures 131: 1028-1039.

https://doi.org/10.1016/j.compstruct.2015.06.043.

18. Rahimi, G. H.; Arefi, M.; Khoshgoftar, M. J. 2012. Electro elastic analysis of a pressurized thick-walled functionally graded piezoelectric cylinder using the first order shear deformation theory and energy method, Mechanika 18 (3): 292-300. https://doi.org/10.5755/j01.mech.18.3.1875.

19. Nejad, M. Z.; Abedi, M.; Lotfian, M. H.; Ghannad, M. 2014. Meshless analysis of cracked functionally graded materials under thermal shock, Mechanika 20 (3): 254-258.

https://doi.org/10.5755/j01.mech.20.3.7395.

20. Farzam-Rad, S. A.; Hassani, B.; Karamodin, A. 2017. 
Isogeometric analysis of functionally graded plates using a new quasi-3D shear deformation theory based on physical neutral surface, Composites Part B-Engineering 108: 174-89.

https://doi.org/10.1016/j.compositesb.2016.09.029.

21. Xiang, S.; Kang, G. W.; Yang, M. S.; Zhao, Y. 2013. Natural frequencies of sandwich plate with functionally graded face and homogeneous core, Composite Structures 96: 226-31. https://doi.org/10.1016/j.compstruct.2012.09.003.

22. Foraboschi, P. 2012. Analytical model for laminatedglass plate, Composites Part B-Engineering 43 (5): 2094-2106. https://doi.org/10.1016/j.compositesb.2012.03.010.

23. Galuppi, L.; Royer-Carfagni, G. 2014. Buckling of three-layered composite beams with viscoelastic interaction, Composite Structures 107: 512-521. https://doi.org/10.1016/j.compstruct.2013.08.006.

24. Kim, J.; Sholar, G. A.; Kim, S. 2008. Determination of accurate creep compliance and relaxation modulus at a single temperature for viscoelastic solids, Journal of Materials in Civil Engineering 20 (2): 147-156. https://doi.org/10.1061/(asce)08991561(2008)20:2(147).

25. Kolarik, J.; Pegoretti, A. 2008. Proposal of the Boltzmann-like superposition principle for nonlinear tensile creep of thermoplastics, Polymer Testing 27 (5): 596606. https://doi.org/10.1016/j.polymertesting.2008.03.002.

26. Shukla, A.; Joshi, Y. M. 2017. Boltzmann superposition principle for a time-dependent soft material: assessment under creep flow field, Rheologica Acta 56 (11): 927940. https://doi.org/10.1007/s00397-017-1044-x.

27. Van Duser, A.; Jagota, A.; Bennison, S. J. 1999. Analysis of glass/polyvinyl butyral laminates subjected to uniform pressure, Journal of Engineering MechanicsAsce 125 (4): 435-442.

https://doi.org/10.1061/(asce)07339399(1999)125:4(435).

28. Kapuria, S.; Bhattacharyya, M.; Kumar, A. N. 2008. Bending and free vibration response of layered functionally graded beams: A theoretical model and its experimental validation, Composite Structures 82(3): 390-402. https://doi.org/10.1016/j.compstruct.2007.01.019.

29. Bhattacharyya, M.; Kapuria, S.; Kumar, A. N. 2007. On the stress to strain transfer ratio and elastic deflection behavior for $\mathrm{Al} / \mathrm{SiC}$ functionally graded material, Mechanics of Advanced Materials and Structures 14(4): 295-302.

https://doi.org/10.1080/15376490600817917.

30. Finot, M.; Suresh, S.; Bull, C.; Sampath, S. 1996. Curvature changes during thermal cycling of a compositionally graded Ni-A12O3 multi-layered material, Materials Science and Engineering: A-Structural Materials Properties Microstructure and Processing 205 (1-2): 59-71. https://doi.org/10.1016/0921-5093(95)09892-5.

Z. Y. Yang, P. Wu, W. Q. Liu

\section{TIME-DEPENDENT CHARACTERISTICS OF TWO-LAYER FUNCTIONALLY GRADED PLATES ADHESIVELY BONDED BY A VISCOELASTIC INTERLAYER BASED ON KIRCHHOFF PLATE THEORY}

S u m m a r y

An analytical solution is proposed to investigate the time-dependent characteristics of two-layer functionally graded plates with a viscoelastic interlayer. The elastic modulus in each graded layer varies through the thickness following an arbitrary function, and its mechanical properties are described based on the Kirchhoff theory. The MaxwellWiechert model is applied to simulate the viscoelastic adhesive interlayer with the neglect of memory effect. The energy equation of the system is expressed by the deformation components, which are expanded as the double trigonometric series. By virtue of variational method, the solutions of stress and deformation are determined efficiently. The comparison study indicates that the present solution matches the finite element solution well; however, the finite element method is highly time-consuming because of the fine mesh in the geometric shape and the time step. Finally, the influences of the geometry and material on the time-dependent behavior of the structure are discussed in detail.

Keywords: functionally graded material, viscoelastic interlayer, time-dependent behavior, variational method.

Received January 20, 2020

Accepted December 01, 2020

This article is an Open Access article distributed under the terms and conditions of the Creative Commons Attribution 4.0 (CC BY 4.0) License (http://creativecommons.org/licenses/by/4.0/). 